Editorial

\title{
Catalytic Upgrading of Biorenewables to Value-Added Products
}

\author{
Hu Li ${ }^{1},{ }^{1}$ Masaru Watanabe, ${ }^{2}$ and Shunmugavel Saravanamurugan ${ }^{3}$ \\ ${ }^{1}$ State-Local Joint Engineering Lab for Comprehensive Utilization of Biomass, Center for R\&D of Fine Chemicals, \\ Guizhou University, Guiyang, Guizhou 550025, China \\ ${ }^{2}$ Research Center of Supercritical Fluid Technology, Graduate School of Engineering, Tohoku University, 6-6-11, Aoba, Aramaki, \\ Aoba-ku, Sendai 980-8579, Japan \\ ${ }^{3}$ Laboratory of Bioproduct Chemistry, Center of Innovative and Applied Bioprocessing (CIAB), Mohali 140306, Punjab, India
}

Correspondence should be addressed to Hu Li; hli13@gzu.edu.cn

Received 24 March 2019; Accepted 24 March 2019; Published 2 May 2019

Copyright (c) $2019 \mathrm{Hu} \mathrm{Li}$ et al. This is an open access article distributed under the Creative Commons Attribution License, which permits unrestricted use, distribution, and reproduction in any medium, provided the original work is properly cited.

Lignocellulosic biomass, as a promising candidate, is being developed to be the most abundant and carbon-neutral feedstock for manufacturing petroleum-based commodities through appropriate design of catalytic materials with controllable functionalities or establishment of fitting catalytic processes. This special issue intends to highlight current progress on the development and optimization of catalytic systems and processes for the selective transformation of biorenewables to value-added products. The papers selected are on the application of new and green technologies to upgrade biomass and waste resources, and those with topics on the preparation of functional catalytic materials and the use of correlated auxiliaries to boost reaction rate and selectivity in the production process are also considered. Hereby, we are pleased to share ten exciting papers on biomass valorization with the readers. We would like to appreciate all the authors for submitting their articles and all the reviewers for their excellent feedback.

In the paper entitled "Influence of Ethanol Organosolv Pulping Conditions on Physicochemical Lignin Properties of European Larch," M. Hochegger et al. characterize and assess the potential applicability of the organosolv lignin fraction from European larch sawdust, based on eight different samples prepared under various reaction conditions (reaction temperature: $420-460 \mathrm{~K}$ and sulfuric acid loading: $0.00-1.10 \%)$ with one milled wood lignin sample as reference. The antiradical potential, the chemical structure, and the molecular weight distribution of the isolated lignin exhibit a direct relationship with the examined reaction parameters.

In the paper entitled "Effect of Metal Chlorides on the Pyrolysis of Wheat Straw," Y. V. Lugovoy et al. present the influence of the addition of $10 \mathrm{wt} . \% \mathrm{FeCl}_{3}, \mathrm{CoCl}_{2}, \mathrm{NiCl}_{2}$, $\mathrm{ZnCl}_{2}, \mathrm{SnCl}_{2}$, or $\mathrm{CuCl}_{2}$ on the wheat straw pyrolysis process. Among the investigated metal chlorides, $\mathrm{CuCl}_{2}$ shows the highest influence on the pyrolysis process of wheat straw, which not only results in a decrease in the molecular weight distribution of volatile products but also leads to a decrease in the yield of gaseous pyrolysis products as well as an increase in the specific surface area of the solid pyrolysis residue.

In the paper entitled "Upgrading Bio-Oil Produced from Corn Cobs and Cedrela odorata via Catalytic Olefination and Esterification with 3,7-Dimethyloct-1-ene and Butanol," F. A. Dawodu et al. upgrade bio-oil produced from corn cobs and Cedrela odorata by simultaneous olefination and esterification using 3,7-dimethyl-1-octene and butanol as a reagent and co-solvent, respectively. The upgraded bio-oils reveal a significant reduction in water and oxygen contents and an increase in the high heating value and flammability.

In the paper entitled "One-Step Synthesis of $\mathrm{CaO}-\mathrm{ZnO}$ Efficient Catalyst for Biodiesel Production," J. T. Arana et al. introduce one-step preparation of $\mathrm{CaO}-\mathrm{ZnO}$ microparticles via mixing $\mathrm{ZnO}$ with $\mathrm{CaCO}_{3}$ and subsequent calcination, which is disclosed to have the characteristic crystallographic cubic structure of $\mathrm{CaO}$ and the hexagonal phase of $\mathrm{ZnO}$. A moderate biodiesel yield of $73 \%$ can be obtained from 
soybean oil through transesterification over the heterogeneous $\mathrm{CaO}-\mathrm{ZnO}$ catalyst at $60^{\circ} \mathrm{C}$ after $6 \mathrm{~h}$.

In the paper entitled "Synthesis of a New Copper-Based Supramolecular Catalyst and Its Catalytic Performance for Biodiesel Production," F. Chang et al. prepare a new copperbased supramolecular catalyst from $\mathrm{CuSO}_{4} \cdot 5 \mathrm{H}_{2} \mathrm{O}$ and $\beta$-cyclodextrin $(\beta-\mathrm{CD})$ by simple chemical complex. After reacting at $120^{\circ} \mathrm{C}$ for $9 \mathrm{~h}$, the $\mathrm{Cu}-\beta-\mathrm{CD}$ catalyst affords a high biodiesel yield of $88.6 \%$ from transesterification of Xanthium sibiricum Patr oil. As compared with the sole species copper or $\beta$-CD, the improvement in the activity of the $\mathrm{Cu}$-based catalyst can be attributed to the synergistic catalytic role of $\mathrm{Cu}^{2+}$ and $\beta-\mathrm{CD}$.

In the paper entitled "Carbon-Based Catalyst from Pyrolysis of Waste Tire for Catalytic Ethanol Dehydration to Ethylene and Diethyl Ether," E. Chaichana et al. investigate the utilization of waste tire as a carbon source in the preparation of carbon-based catalysts for ethanol dehydration. The carbon catalyst prepared by treatment with $\mathrm{HCl}$ and calcination at $420^{\circ} \mathrm{C}$ displays superior ethanol conversion of $36.2 \%$ at $400^{\circ} \mathrm{C}$ with selectivity of 65.9 and $33.5 \%$ toward ethylene and diethyl ether, respectively, which can be ascribed to the relatively high surface acid density.

In the paper entitled "Ethanol Dehydration over $\mathrm{WO}_{3} /$ $\mathrm{TiO}_{2}$ Catalysts using Titania Derived from Sol-Gel and Solvothermal Methods," A. Tresatayawed et al. study the catalytic activity of $\mathrm{WO}_{3} / \mathrm{TiO}_{2}$ in the dehydration of ethanol to value-added products including ethylene, diethyl ether, and acetaldehyde. The preparation methods (i.e., the sol-gel and solvothermal approach) are found to essentially alter the physicochemical properties of $\mathrm{TiO}_{2}$ supports, where the pore structure, acidity, and $\mathrm{WO}_{3}$ distribution of the catalysts directly affect the reactivity and product selectivity. The $\mathrm{WO}_{3} / \mathrm{TiO}_{2}$ catalyst prepared by the solvothermal method with high acidity exhibits the highest ethanol conversion (ca. $88 \%)$ at $400^{\circ} \mathrm{C}$, while the presence of $\mathrm{WO}_{3}$ offers a remarkable increase in diethyl ether selectivity (ca. 68\%) at $250^{\circ} \mathrm{C}$.

In the paper entitled "Catalytic Transfer of Fructose to 5Hydroxymethylfurfural over Bimetal Oxide Catalysts," Q. Zhang et al. modify aluminum-molybdenum mixed oxide with stearic acid to be prominent solid acid catalysts for the direct conversion of sugars to 5-hydroxymethylfurfural (HMF). A high HMF of $49.8 \%$ is obtained from fructose by dehydration, with moderate HMF yields of $24.9 \%$ and $27.6 \%$ being attained from glucose and sucrose, respectively. The good activity and reusability of the catalyst can be resulted from its sufficient acidic site, mesoporous structure, high surface area, and good stability.

In the paper entitled "Upgrading of Carbohydrates to the Biofuel Candidate 5-Ethoxymethylfurfural (EMF)," X. Liu and $\mathrm{R}$. Wang review the reaction performance of various catalysts (e.g., mineral salts, zeolites, heteropolyacid-based hybrids, sulfonic acid-functionalized materials, and ionic liquids) in the selective conversion of hexose sugars to EMF, providing potential strategies and directions for the design of novel catalytic materials and systems to further improve the yield and selectivity toward EMF.
In the paper entitled "Chemocatalytic Production of Lactates from Biomass-Derived Sugars," H. Zhang et al. comment on the state of the art for the synthesis of lactic acid and its esters from sugars and real biomass like rice straw catalyzed by homogeneous and heterogeneous acids and bases. Emphasis is placed on the advantages of heterogeneous catalytic systems, and suggestions on the improvement of their catalytic reactivity in the production of lactic acid are given thereof.

\section{Conflicts of Interest}

The editors declare that they have no conflicts of interest.

$\mathrm{Hu} \mathrm{Li}$

Masaru Watanabe

Shunmugavel Saravanamurugan 


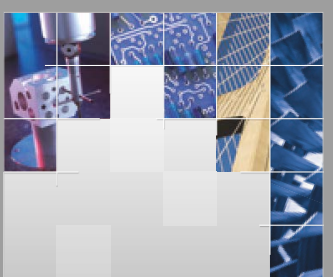

\section{Enfincering}
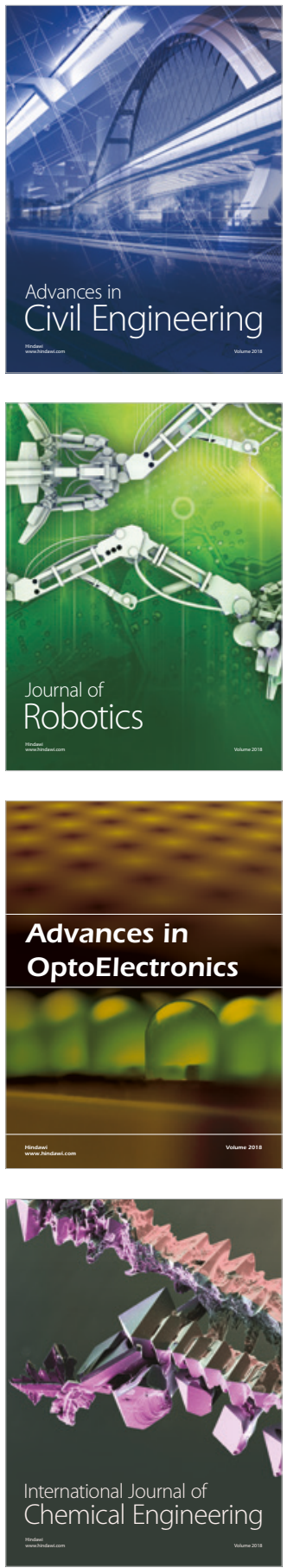

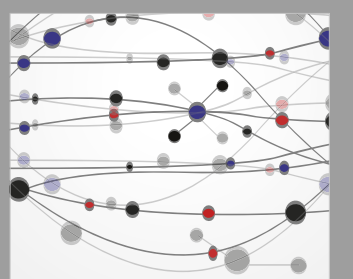

\section{Rotating \\ Machinery}

The Scientific World Journal

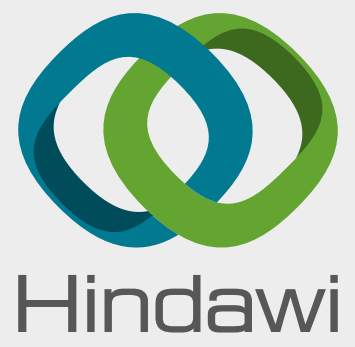

Submit your manuscripts at

www.hindawi.com
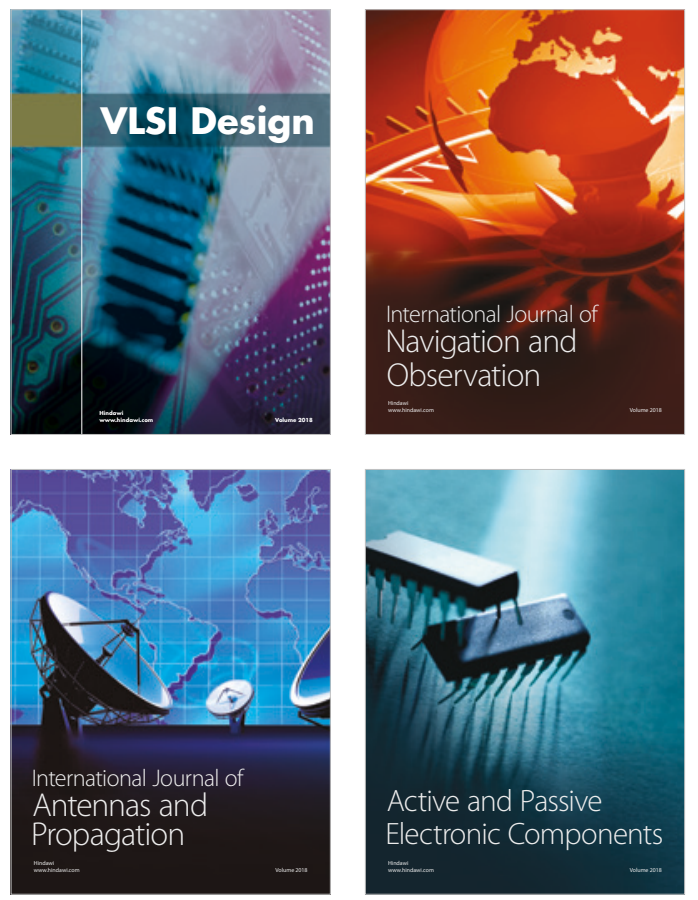
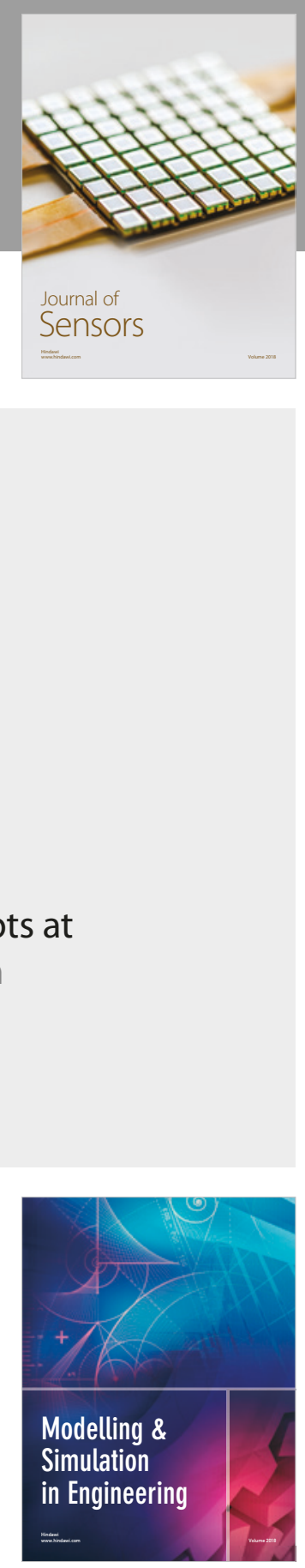

\section{Advances \\ Multimedia}
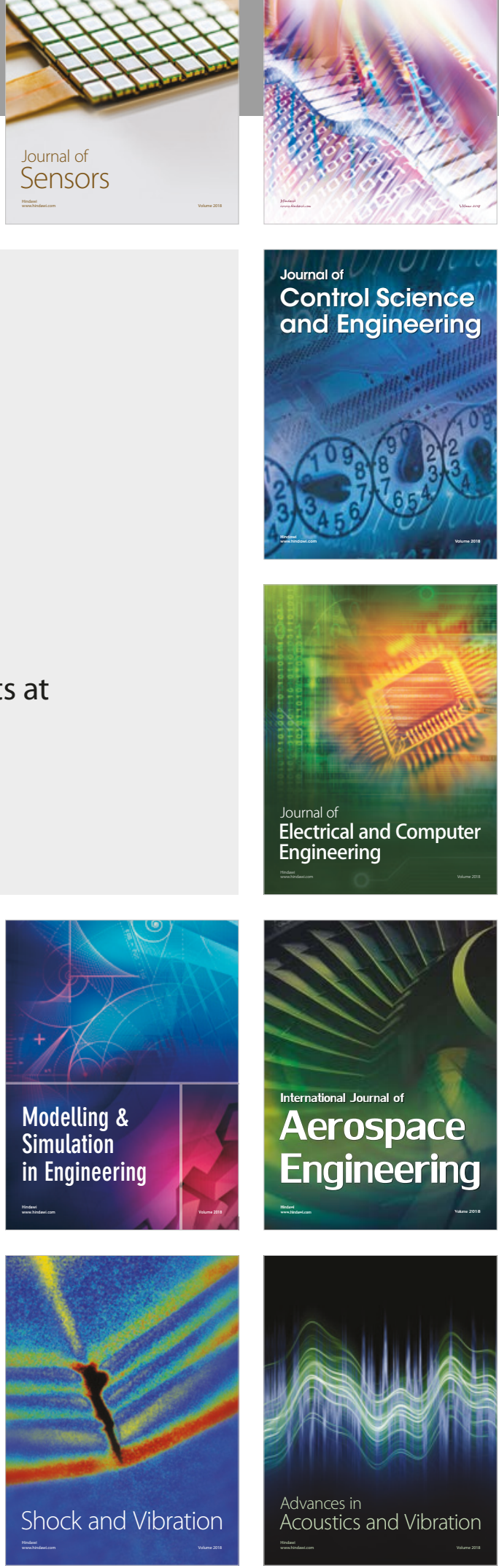